\title{
A group size effect on personal risk judgments: Implications for unrealistic optimism
}

\author{
PAUL C. PRICE \\ California State University, Fresno, California
}

\begin{abstract}
In Experiments 1 and 2, college students ( $N=32$ and $N=18$, respectively)read heart attack risk profiles (i.e., lists of risk factors) for each of several employees at a series of fictional companies and judged the heart attack risk of the typical employee at each company. In both experiments, subjects' risk judgments increased as a function of the number of employees at the companies. In Experiments $3 \mathrm{~A}$ and 3B, college students ( $N=56$ and $N=33$, respectively) judged the heart attack risk of the typical employee at a company and also judged the risk of each individual employee. In these experiments, the typical employee was generally judged to be at higher risk than the individual employees. This group size effect might help to explain unrealistic optimism - people's tendency to judge themselves to be at lower risk than their peers for negative life events. Furthermore, it can be modeled successfully within Fiedler's (1996) BIAS framework.
\end{abstract}

People must often judge their own risk, or someone else's risk, of experiencing negative life events (Weinstein, 1980). It is important to understand such personal risk judgments in part because many consequential decisions might be based on them. For example, the decision to engage in a health-protective behavior like eating a low-fat diet might be based to some extent on a person's judgment of his/her risk of developing heart disease or other diet-related health problems (Janz \& Becker, 1984). Despite their importance, however, the psychological processes underlying personal risk judgments are not well understood.

The present research was conducted, therefore, to develop and test a new hypothesis about personal risk judgments. The new hypothesis is that there is a group size effect on people's personal risk judgments, such that the typical member of a larger group is generally judged to be at higher risk than the typical member of a smaller group. The significance of the group size effect is twofold. First, it might help to explain unrealistic optimism: people's pervasive tendency to judge themselves to be at lower risk than their peers for various negative life events (Weinstein, 1980). Second, it can be modeled successfully within Fiedler's (1996) BIAS framework for understanding social judgment, which immediately suggests several new hypotheses about personal risk judgments and the psychological processes underlying them. The specific model presented here assumes that people form memory representations of the individuals they encounter, encoding the high-risk features of those individuals more effi-

This research was supported in part by a grant from the Decision, Risk, and Management Science Program, National Science Foundation. Kang Hang deserves thanks for collecting most of the data presented here. Correspondence should be addressed to P. C. Price, Department of Psychology, California State University, Fresno, 5310 North Campus Drive, Fresno, CA 93740-8019(e-mail: paulpri@ csufresno.edu). ciently than their low-risk features. At the time of judgment, the memory representations of the individuals in the target group are averaged, and the risk judgment is based on the similarity of this average to a high-risk prototype.

In the following, the empirical and theoretical rationale for expecting a group size effect on people's personal risk judgments is presented, beginning with the observation that people tend to judge themselves to be at lower risk than their peers for various negative life events. Then, three experiments are presented, all of which clearly confirm the existence of a group size effect on people's personal risk judgments in a controlled laboratory paradigm. Finally, the implications of the group size effect for understanding unrealistic optimism are reviewed and the BIAS model is described.

A robust result from research on personal risk judgments is that people tend to judge their own risk to be lower than that of their peers (Weinstein, 1980). This phenomenon, sometimes referred to as unrealistic optimism, has been observed for judgments about health risks like developing AIDS and cancer (Bauman \& Siegel, 1987; Weinstein, 1980), accident risks like being injured in a car crash and while bungee jumping (McKenna, 1993; Middleton, Harris, \& Surman, 1996; Svenson, 1980), and social risks like being fired from a job and getting divorced (Baker \& Emery, 1993; Klar, Medding, \& Sarel, 1996; Weinstein, 1980). A variety of explanations have been proposed to account for this phenomenon, many of which are motivational. That is, it is often assumed that people judge their own risk to be lower than that of their peers either to reduce anxiety or to preserve self-esteem (BoneyMcCoy, Gibbons, \& Gerrard, 1999; Perloff \& Fetzer, 1986; Taylor, 1989; Weinstein, 1980). Other explanations, however, have emphasized cognitive factors-typically qualitative differences in how people process information about themselves as opposed to others (McKenna, 1993) 
or how they process information about individuals as opposed to groups (Klar et al., 1996). Although many of these explanations have received some empirical support, it is clear that none of them provides a complete account of unrealistic optimism (Weinstein \& Klein, 1996).

One theoretical possibility, which has yet to be considered, is that unrealistic optimism is a special case of a more general group size effect on people's personal risk judgments. Note that a risk judgment for oneself differs from a risk judgment for one's peers not only in that the former concerns oneself and the latter concerns one's peers, but also in that the former concerns a group of size $N=1$ and the latter concerns a group of size $N>1$. If there were a general tendency to judge the average or typical member of a larger group to be at higher risk than the average or typical member of a smaller group, then unrealistic optimism would be a straightforward implication.

There are additional reasons-both empirical and theoretical-for expecting such a group size effect. First, a number of researchers have shown that the magnitude of unrealistic optimism depends on the nature of the target group with which people compare themselves (Klein \& Weinstein, 1997). For example, Perloff and Fetzer (1986) found that college students judged the risk for the average student and the average person to be greater than that of their closest friend, same-sex parent, and a sibling. Furthermore, subjects' judgments for the latter three targets did not differ significantly from their judgments of their own risk (see also Whitley \& Hern, 1991). Klar et al. (1996) have shown that this basic effect is observed even when the comparison individual is not a friend or relative of the subject. For example, they found that unrealistic optimism was essentially eliminated when subjects were asked to compare their own risk to that of an acquaintance or to that of a randomly selected individual sitting in the same classroom. Harris and Middleton (1994) obtained similar results, but they also found slight differences for different target individuals (oneself, a friend's friend, and an acquaintance; see also Regan, Snyder, \& Kassin, 1995).

This basic pattern of results can be interpreted as a general group size effect. As the size of the comparison target group increases, the judged risk of the typical member of the target group increases too. None of these researchers, however, has interpreted their results in this way. Perloff and Fetzer (1986) proposed a variety of motivational and cognitive mechanisms. First, they suggested that people might be motivated to judge individuals who are close to them (e.g., their best friend) to be at relatively low risk. Second, they suggested that a vaguely specified target group might allow people greater leeway in choosing a specific individual for comparison. They might, therefore, choose a high-risk comparison individual to make themselves appear to be at relatively low risk (see also Harris \& Middleton, 1994). Third, they suggested that asking people to judge the risk of the average target group member might serve as a cue to retrieve from memory an exemplar of an extremely high risk individual, which then serves as the comparison (see also Whitley \& Hern, 1991). Klar et al. (1996) proposed that whereas people think about individuals in a singular mode, considering mainly their risk-reducing characteristics and behaviors, they think about groups in a distributional mode, considering mainly statistical information such as the relative frequency of the negative event. One important goal of the present research, therefore, was to demonstrate a group size effect on personal risk judgments while minimizing the number of alternative interpretations.

Theoretically, the existence of a group size effect is consistent with a number of interrelated models of memory and judgment. Specifically, many exemplar-based models, in which knowledge about a group is contained in a set of exemplars (representations of the individuals who make up that group), predict group size effects under some conditions (Dougherty, Gettys, \& Ogden, 1999; Fiedler, 1996; Smith, 1991; Smith \& Zaraté, 1992). Consider, for example, the phenomenon of illusory correlation, in which people perceive a statistical association between the larger of two social groups and the more frequent of two types of behavior, even when there is no objective association between these variables (Hamilton \& Gifford, 1976). In essence, this is a group size effect, which both Fiedler (1996) and Smith (1991) have shown can be explained within an exemplar-based framework. Again, a specific model of personal risk judgments based on Fiedler's BIAS framework is presented in the General Discussion.

In contrast with subjects in previous research on personal risk judgments, subjects in the present experiments performed a laboratory task in which they were presented with risk factor information about each member of a target group and then judged the risk of the typical member of that target group. The advantage of this approach is that it allows for both the systematic manipulation of target group size and the careful control of potential confounding variables. The ability to manipulate target group size over a range of values is important because theories such as that of Klar et al. (1996; see also Alicke, Klotz, Breitenbecher, Yurak, \& Vredenburg, 1995) imply that there should be a sharp difference in the perceived risk of target groups of size $N=1$ and all other target groups. The hypothesis of a general group size effect, however, implies a continuous increase in perceived risk as a function of target group size. The ability to control potential confounding variables is important because motivational theories assume that the closeness or perceived similarity of the target group to oneself is the key independent variable (e.g., Boney-McCoy et al., 1999; Perloff \& Fetzer, 1986; Taylor, 1989; Weinstein, 1980). The present hypothesis, however, implies that target group size has an effect on perceived risk independent of these other variables.

\section{EXPERIMENT 1}

Subjects were presented with the "heart attack risk profiles" of employees at two small companies $(n=6)$ and two large companies $(n=12)$ and asked to judge the heart attack risk of the typical employee at each com- 
pany. If there is a group size effect, then the typical employee at the large companies should be judged to be at higher risk than the typical employee at the small companies. The objective risk of the employees was also manipulated so that one of the small companies and one of the large companies consisted primarily of low-risk employees, and the other small and large companies consisted primarily of high-risk employees. This manipulation was included because it has been suggested that the magnitude of the group size effect, and even its direction, might depend on the objective risk of the target group members (Price, Lee, \& Voth, 1999).

\section{Method}

\section{Subjects}

The subjects were 32 students at California State University, Fresno. They participated as part of an introductory psychology course requirement.

\section{Design and Procedure}

The subjects' task was to read information about each of the employees at each of four fictional companies and to judge the heart attack risk of the typical employee at each company. Each employee was identified by his/her initials and was described in terms of eight binary risk factors for heart attack and stroke (American Heart Association, 1998). Figure 1 shows how this information might have been displayed for 1 employee. The employees were organized into four fictional companies. Two of the companies were relatively small, consisting of 6 employees each, and the other two were relatively large, consisting of 12 employees each. One small company and one large company were low-risk companies, and the other small and large companies were high-risk companies. The stimuli were generated randomly on a trial-by-trial basis in the following way. For employees in the low-risk companies, each risk factor had a probability of .25 of taking the high-risk value (e.g., male, over 45 , etc.). For employees in the high-risk companies, each risk factor had a probability of .75 of taking the high-risk value. Figure 2 shows the resulting distributions of the number of high-risk values

Employee: K.H.

$\begin{array}{ll}\text { Sex: } & \text { Female } \\ \text { Age: } & \text { Over 45 } \\ \text { Family History: } & \text { None } \\ \text { Smokes Cigarettes: } & \text { No } \\ \text { Blood Pressure: } & \text { High } \\ \text { Cholesterol Level: } & \text { Good } \\ \text { Regular Exercise: } & \text { No } \\ \text { Weight: } & \text { Good }\end{array}$

When you are ready to continue, press the return key.

Figure 1. Sample stimulus display for a single employee.

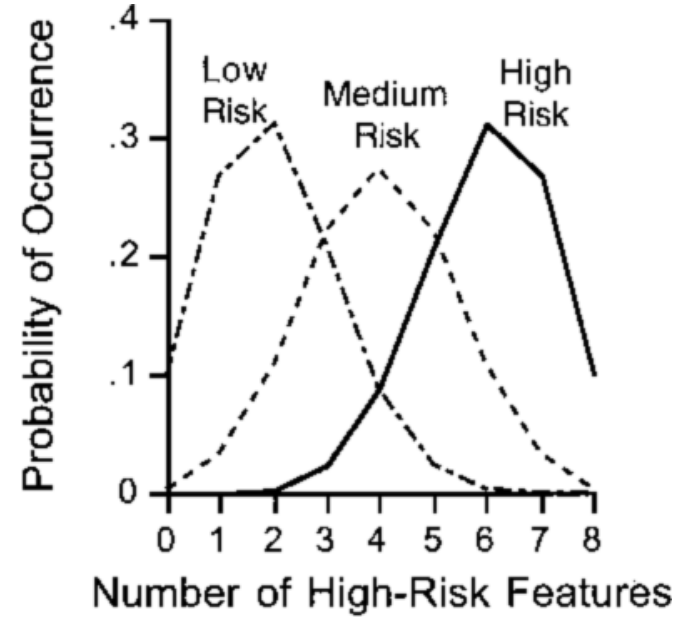

Figure 2. Distribution of the number of high-risk features across stimulus employees in low-, medium-, and high-risk companies.

across employees for both the high- and low-risk companies (and for a medium-risk company; see Experiment 2). Note that these distributions essentially guaranteed that low-risk companies and highrisk companies consisted almost exclusively of low-risk and highrisk employees, respectively.

The subjects were tested individually. The stimuli were presented and responses collected using desktop computers. Subjects began by reading a set of instructions that described their task in a general way, presented examples of extremely high- and low-risk employees, and displayed the rating scale they would eventually use to make their risk judgments. Then they observed the employees at the first company. The presentation of the employees was self-paced, and most subjects spent approximately 10-20 sec per employee. After observing the last employee at that company, subjects rated the heart attack risk of the typical employee on a 7-point rating scale. The rating scale was displayed as a horizontal line with the numerals 1-7 displayed at equal intervals above the line from left to right. Below the line were displayed a set of verbal labels to accompany the numerical ratings: extremely low, very low, somewhat low, moderate, somewhat high, very high, and extremely high. Subjects responded by typing a number from 1 to 7 . Then they proceeded to observe the employees at the second company, make their risk judgments, and so on. The order in which the four companies were presented was randomized for each subject.

\section{Results and Discussion}

The means of subjects' heart attack risk judgments for the four companies are presented in Figure 3. Not surprisingly, the typical employee at the high-risk companies was judged to be at higher risk than the typical employee at the low-risk companies. In addition, the typical employee at the large companies was judged to be at higher risk than the typical employee at the small companies. To confirm these observations statistically, a repeated measures ANOVA was conducted on the heart attack risk ratings, with objective risk and company size as within-subjects factors. There were main effects of both objective risk $\left[F(1,31)=184.40, M S_{\mathrm{e}}=1.02, p<.05\right]$ and company size $\left[F(1,31)=5.10, M S_{\mathrm{e}}=0.81, p<.05\right]$, but there was no interaction between these two factors $\left[F(1,31)=0.36, M S_{\mathrm{e}}=1.06\right.$, n.s. $]$. In other words, there 


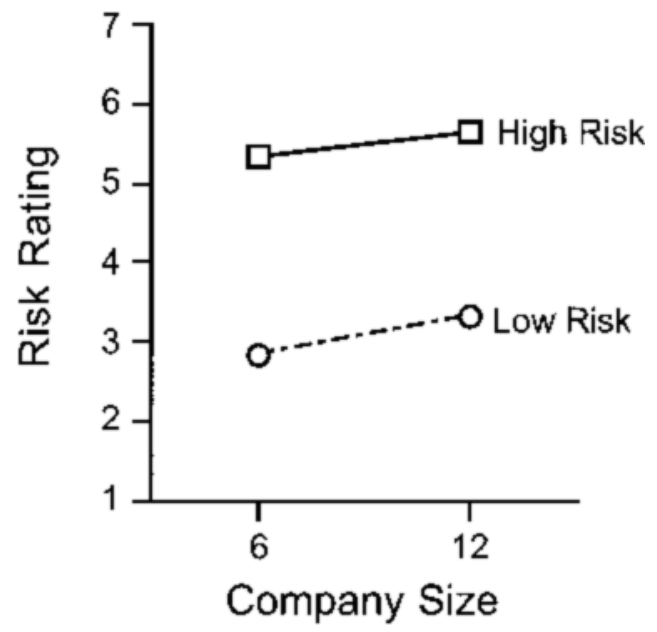

Figure 3. Mean heart attack risk ratings as a function of objective risk and company size for Experiment 1.

was indeed a group size effect on subjects' risk judgments, which was consistent across the different objective risk levels. To be more precise, each additional employee increased subjects' risk ratings by about .06 rating scale points. Although this seems like a small effect, it may be sufficient to account for much of the standard unrealistic optimism effect because the comparison target in the typical study is a very large group (e.g., the students at one's college or university). Note also that this group size effect cannot readily be explained as a motivational phenomenon because there is no reason to think that subjects were motivated to judge the typical employee at a 6-person company to be at lower risk than the typical employee at a 12-person company. Nor can it be readily explained by theories that assume a difference in how people process information about themselves as opposed to others or a difference in how people process information about individuals as opposed to groups, because these variables were controlled. The targets were always others and they were always groups. These results lend support to the idea that the phenomenon of unrealistic optimism could be, in part, a group size effect.

\section{EXPERIMENT 2}

Again, because the typical unrealistic optimism experiment has involved a comparison between a small target group (consisting of oneself) and a large target group (consisting of one's peers), it could be that unrealistic optimism reflects, in part, the group size effect demonstrated here. This assumes, of course, that the group size effect demonstrated here extends to groups of size $N=1$. It could still be, however, that people think differently about target groups of size $N=1$ than about target groups of size $N>1$, and therefore the judged risk of the typical member of a target group of size $N=1 \mathrm{might}$ not follow the same trend displayed in Figure 3. For this reason it is important to demonstrate the group size effect for target groups of size $N=1$, which was the main goal of Experiment 2.

\section{Method}

\section{Subjects}

The subjects were 18 undergraduate students at California State University, Fresno. They participated as part of an introductory psychology course requirement.

\section{Design and Procedure}

The design and procedure were similar to those of Experiment 1. This time, however, there were three different company sizes and three different objective risk levels. In the small, medium, and large companies, there were 1, 5, and 9 employees, respectively. For employees in the low-, medium-, and high-risk companies, the probability that each risk factor would take the high-risk value was .25 , .50 , and .75 , respectively. Figure 2 shows the distribution of the number of high-risk values across the employees of the low-, medium-, and high-risk companies. Company size was again a withinsubjects factor, but objective risk was now a between-subjects factor. That is, each subject judged the heart attack risk of the typical employee at each of three companies: a small, medium, and large company at which the employees tended to be at low, medium, or high risk. Subjects were randomly assigned to one of the three objective risk levels, and the order in which the small, medium, and large companies were presented was randomized for each subject. The instructions again described the subjects' task in a general way, but this time omitted the presentation of the actual response scale. In making their risk judgments, subjects clicked on one of seven buttons representing the possible responses on the horizontally oriented rating scale.

\section{Results and Discussion}

The means and standard deviations of subjects' heart attack risk ratings are presented in Figure 4. Again, not surprisingly, there was a statistically significant main effect of objective risk level $\left[F(2,15)=12.34, M S_{\mathrm{e}}=0.64\right.$, $p<.05]$. There was also a statistically significant main effect of company size $\left[F(2,30)=7.09, M S_{\mathrm{e}}=0.97, p<\right.$ $.05]$, replicating the group size effect. Also as in Experiment 1, there was no statistically significant interaction

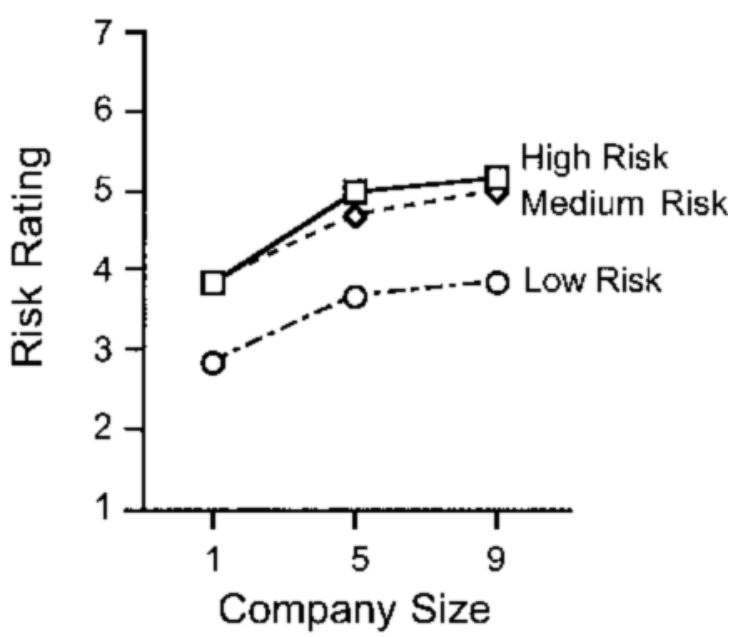

Figure 4. Mean heart attack risk ratings as a function of objective risk and company size for Experiment 2. 
between these two factors $[F(4,30)=0.08$, n.s.]. It also appears from Figure 2 that the group size effect demonstrated in Experiment 1 extends in a relatively straightforward way to target groups of size $N=1$. Note, however, that the increase in company size from small to medium increased the risk rating by approximately 0.26 rating scale points per additional employee, whereas the increase from medium to large increased the risk rating by approximately 0.04 rating scale points per additional employee. Although this could indicate that there is a qualitative difference between how people think about individuals and how they think about groups, this result is also a straightforward implication of the BIAS model, to be considered in the General Discussion.

\section{EXPERIMENT 3}

An interesting implication of the group size effect demonstrated in Experiments 1 and 2 is that the individuals who make up a group should generally be judged at lower risk than the typical member of that group. In a sense, Experiment 2 demonstrated this because the individual employees at the companies of size $N=1$ were drawn from the same population as the individual employees at the companies of size $N>1$. Experiment 3 was conducted to test this implication more directly, however, by having subjects judge the heart attack risk of the typical employee at a company of size $N=10$ and then judge the heart attack risk of the same 10 employees as individuals. Experiment 3A employed a within-subjects design. All subjects judged the risk of the typical employee before judging the risk of the individual employees because it has been shown that people are fairly good at estimating the mean of a series of numbers (Peterson \& Beach, 1967). Had they judged the risk of the individual employees first, they might simply have averaged their judgments about the individuals in making their judgments about the typical employee. Clearly, such a strategy would not be expected to lead to a group size effect. To guard against the possibility of order effects, therefore, Experiment 3B was conducted as a replication of Experiment $3 \mathrm{~A}$ using a different set of 10 heart attack risk profiles that had already been judged as individuals in a pilot study. Thus, Experiment 3B also allowed a betweensubjects comparison of one group's judgments of the risk of the typical employee with another group's judgments of the risk of the individual employees.

\section{Method}

\section{Subjects}

The subjects were 122 undergraduate students at California State University, Fresno. Fifty-six participated in Experiment 3A, 33 participated in Experiment 3B, and 33 participated in the pilot study that provided the comparison group for Experiment 3B. All subjects participated as part of an introductory psychology course requirement.

\section{Procedure}

Pilot study. Thirty-three students were tested in a single classroom session. Each one rated the risk of each of a set of 24 fictional individuals who varied widely in terms of their objective risk. The 24 risk profiles were presented in a booklet, in a fixed order, with six risk profiles on each page. Below each risk profile was printed the 7-point rating scale used in Experiments 1 and 2. Participants were instructed to rate the heart attack risk of each individual by circling one of the numbers $1-7$ on the rating scale.

Experiment 3A. Ten heart attack risk profiles were created, each of which had from one to four high-risk features, with a mean of 2.90 and a standard deviation of 0.59 . Each risk prof ile was then copied onto an overhead transparency. The 56 subjects were tested in a single classroom session. During the first part of the experiment, the 10 heart attack risk profiles were presented to subjects one at a time for approximately $15 \mathrm{sec}$ each, with an interval of $2-3 \mathrm{sec}$ between them. Then the rating scale used in Experiments 1 and 2 was presented on an overhead transparency and subjects were asked to rate the heart attack risk of the typical employee at the company by writing a number from 1 to 7 on a response sheet. During the second part of the experiment, the same 10 heart attack risk profiles were presented to subjects one at a time for approximately $15 \mathrm{sec}$ each, with an interval of $2-3 \mathrm{sec}$ between them. During this part of the experiment, however, the rating scale was displayed continuously and the subjects were asked to rate the heart attack risk of each individual employee by writing a number from 1 to 7 for each one on their response sheet.

Experiment 3B. The design and procedure of Experiment 3A were employed again, but the 10 heart attack risk profiles were chosen from among the 24 that had already been rated by subjects in the pilot study. These 10 heart attack risk profiles were chosen because the mean rating assigned to each of them was between 3 and 5 on the 7-point response scale, and, therefore, the overall mean was close to the midpoint of the scale (i.e., close to 4). They had from two to five high-risk features, with a mean of 3.5 and a standard deviation of 1.02 .

\section{Results and Discussion}

\section{Experiment 3A}

Subjects' ratings of the heart attack risk of the individual employees were compared with their ratings of the heart attack risk for the typical employee. A dependent-samples $t$ test indicated that the mean rating for the individual employees $(3.51, S D=0.78)$ was significantly lower than the mean rating for the typical employee [3.95, $S D=0.84 ; t(55)=3.97, p<.05]$. This is precisely the result implied by the group size effect demonstrated in Experiments 1 and 2 . Note also that across all 56 subjects, the heart attack risk of an individual employee was judged to be lower than that of the typical employee $46 \%$ of the time, the same as that of the typical employee $32 \%$ of the time, and higher than that of the typical employee only $22 \%$ of the time. A dependent-samples $t$ test comparing the mean proportion of individual employees judged to be at lower risk than the typical employee with the mean proportion of individual employees judged to be at higher risk revealed a statistically significant difference $[t(55)=$ $3.83, p<.05]$. Furthermore, this result did not arise because the distributions of subjects' risk judgments for the individual employees were positively skewed (which would, in fact, have meant that more individual employees were below average than above average). The difference was statistically significant both for subjects whose distributions were negatively skewed and for subjects whose distributions were positively skewed. 


\section{Experiment 3B}

Again, subjects' ratings of the heart attack risk of the individual employees at the company were compared with their ratings of the heart attack risk of the typical employee. A dependent-samples $t$ test indicated that the mean risk rating assigned to the individual employees (3.94, $S D=0.66)$ was significantly lower than the mean rating assigned to the typical employee $(4.63, S D=0.82$; $t(32)=4.64, p<.05]$. Also, across all 33 subjects, the heart attack risk of an individual employee was judged to be lower than that of the typical employee $55 \%$ of the time, the same as that of the typical employee $27 \%$ of the time, and higher than that of the typical employee only $18 \%$ of the time. A dependent-samples $t$ test comparing the mean proportion of individual employees judged to be at lower risk than the typical employee with the mean proportion of individual employees judged to be at higher risk revealed a statistically significant difference $[t(32)=$ $4.44, p<.05]$. Again, this difference was statistically significant both for subjects whose distributions of risk ratings for the individuals were negatively skewed and for those whose distributions were positively skewed.

The pilot subjects' mean ratings of the heart attack risk of the individual employees were also compared with the subjects' ratings of the heart attack risk of the typical employee. An independent-samples $t$ test indicated that the mean heart attack risk rating assigned to the individual employees by the pilot subjects $(4.15, S D=0.52)$ was lower than the mean heart attack risk rating assigned to the typical employee by the subjects in Experiment 3B $[t(64)=2.89, p<.05]$. Thus, this between-subjects comparison shows that this result is not due to an order effect.

It is important to consider another possible artifactual explanation for these results. There is a sense in which judgments about individual employees and judgments about the typical employee are not directly comparable. Consider 3 employees who are judged, as individuals, to have heart attack risks of 2,3 , and 5. On average, these employees have a heart attack risk of 3.33. In judging the risk of the typical employee, however, subjects are forced to make an integer response. If they have a tendency to round up rather than down (e.g., to 4 rather than to 3 ), then they will also tend to judge the risk of the group to be greater than the mean risk of the individuals. Note, however, this artifactual explanation cannot be applied to the difference between subjects' risk judgments for companies of size $N=6$ and $N=12$ in Experiment 1 and companies of size $N=5$ and $N=9$ in Experiment 2. It seems much more parsimonious to explain the entire pattern of results as a single group size effect.

\section{GENERAL DISCUSSION}

The experiments described here revealed a consistent group size effect on people's personal risk judgments. The typical member of a larger target group was judged to be at higher risk than the typical member of a smaller target group, even when the objective risk of the individ- uals who made up the groups was the same. This effect seems to extend in a straightforward way to target groups of size $N=1$, such that the individuals who make up a group are generally judged to be at lower risk than the typical member of the group. This result, by itself, implies the unrealistic optimism that has been observed so often in research on people's personal risk judgments (e.g., Weinstein, 1980). Further research is needed, however, to show that this group size effect is a contributor to unrealistic optimism. A first step would be to show that in the standard unrealistic optimism paradigm, the size of the group to which individuals compare themselves influences the magnitude of the bias. Do people judge themselves to be at lower risk relative to the average member of a large group (e.g., the students at their university) than relative to the average member of a small group (e.g., the students in their psychology class)? Although some existing research is consistent with this hypothesis (e.g., Whitley \& Hern, 1991), confounding variables, such as the perceived closeness or similarity of the target group to oneself, need to be adequately controlled.

It is also important to consider theoretical models of the group size effect, both to provide a deeper understanding of it and to guide further research. First, note that motivational theories do not seem to account for the present results. It is unclear why people would be more motivated to judge the typical member of a 6-person group to be at lower risk than the typical member of a 12-person group. A similar argument can be made against all theories that imply a sharp distinction between people's risk judgments about target groups of size $N=1$ and their risk judgments about all larger target groups. This includes the cognitive theory of Klar et al. (1996), according to which people think in a qualitatively different way about individuals and groups.

What is needed, therefore, is a model that uses a small set of related principles to account for the group size effect across the entire range of group sizes used in the present experiments. Ideally, it should include minimal assumptions about subject motivations, perceived similarity, and other variables that were reasonably well controlled in the present experiments. In the following, just such a model is presented. The model-which is based on Fiedler's (1996) BIAS framework for understanding social judgment - belongs to a class of exemplar-based theoretical approaches that has been quite successful in explaining various aspects of memory and judgment (Dougherty et al., 1999; Hintzman, 1986, 1988), including group size effects in other contexts (Fiedler, 1996; Smith, 1991).

The BIAS framework consists of three assumptions, with one especially important implication (Fiedler, 1996). The first assumption is that social judgments are typically based on several multidimensional stimulus observations. In the context of the present experiments, each employee can be conceptualized as a stimulus observation consisting of eight risk-factor dimensions or values. To make this idea concrete, Figure 5 presents a matrix of five stimulus observations, representing the employees 


\section{High-Risk
Risk Factors $\quad$ Prototype}

Employees

Average

Employee

\section{1}

2

3

4

5

Sex

Age

Family History

Smokes Cigarettes

Blood Pressure

Cholesterol Level

Regular Exercise

Weight

\begin{tabular}{|c|c|c|c|c|c|c|}
\hline Male & Male & Female & Male & Female & Male & $\begin{array}{c}\text { Mostly } \\
\text { Male }\end{array}$ \\
\hline$>45$ & $>45$ & $<45$ & $>45$ & $<45$ & $>45$ & $\begin{array}{c}\text { Mostly } \\
>45\end{array}$ \\
\hline Yes & No & Yes & No & No & No & $\begin{array}{c}\text { Mostly } \\
\text { No }\end{array}$ \\
\hline Yes & No & No & No & No & No & No \\
\hline High & High & Good & High & High & Good & $\begin{array}{c}\text { Mostly } \\
\text { High }\end{array}$ \\
\hline High & High & Good & High & Good & Good & $\begin{array}{c}\text { Mostly } \\
\text { Good }\end{array}$ \\
\hline No & Yes & Yes & Yes & Yes & Yes & Yes \\
\hline Over & Over & Good & Good & Good & Over & $\begin{array}{c}\text { Mostly } \\
\text { Good }\end{array}$ \\
\hline
\end{tabular}

Figure 5. BIAS representation of the information on which a heart attack risk rating might be based, including a high-risk prototype, stimulus observations of 5 employees, and an informal average of those five stimulus observations.

at a company of size $N=5$. The second assumption is that social judgments are typically based on an average (or sum) of these stimulus observations. Note that Figure 5 also presents a representation of the average employee, consisting of eight informally averaged risk factor values. The third assumption is that social judgments are made by comparing the average stimulus observation with a standard representation or prototype. For present purposes, it can be assumed that the average stimulus observation is compared with the prototype of an extremely high-risk employee (also represented in Figure 5). Furthermore, to the extent that the average is similar to the prototype, the typical employee is judged to be at high risk.

An important implication of these assumptions holds when the stimulus observations are essentially random variants of an underlying prototype. The greater the number of stimulus observations averaged, the more similar the average stimulus observation is to the underlying prototype. This is essentially an effect of the law of large numbers. For example, the individual employees at a highrisk company can be conceptualized as random variants of a high-risk prototype (i.e., an individual with all highrisk feature values). As the number of employees increases, therefore, the more closely the average employee resembles the high-risk prototype. As a result, employees at larger companies are judged to be at higher risk.

Of course, given these assumptions, one would expect very different results for judgments about the typical employee at a low-risk company. Because the employees at a low-risk company are essentially random variants of a low-risk prototype, one would expect that the typical employee at a large company would be judged to be at lower risk than the typical employee at a small company (Price et al., 1999). The present results, however, revealed no interaction between objective risk and company size. This result can be accommodated within the BIAS framework by assuming that high-risk cue values are more likely to be encoded in memory than are low-risk cue values. This makes sense because subjects knew that the task concerned their perceptions and judgments of risk. Also, high-risk cue values might have been more salient because they represent departures from the generally more common low-risk values (e.g., most people are not overweight). Such selective encoding would produce risk judgments consistent with the results observed here.

Figure 6 presents the results of a BIAS simulation of Experiment 2, which incorporates these assumptions in a rather straightforward way (see the Appendix for details). Note that the $y$-axis of Figure 2 is actually the correlation between the average of the individual employee representations at a target company and the prototype of an extremely high-risk individual. Higher values on the $y$-axis, therefore, correspond to higher risk judgments on the 7-point risk-rating scale. Note that the basic pattern of results from Experiment 2 is replicated by the simulation. There are main effects of both objective risk and company size, but relatively little effect of the interaction between these two factors. Furthermore, the effects of both objective risk and company size appear to be negatively accelerated, just as in Experiment 2.

It is also possible that selective retrieval, rather than selective encoding, is responsible for the group size effect. In fact, Fiedler (1996) has presented a more sophisticated general model in which stimulus observations are 


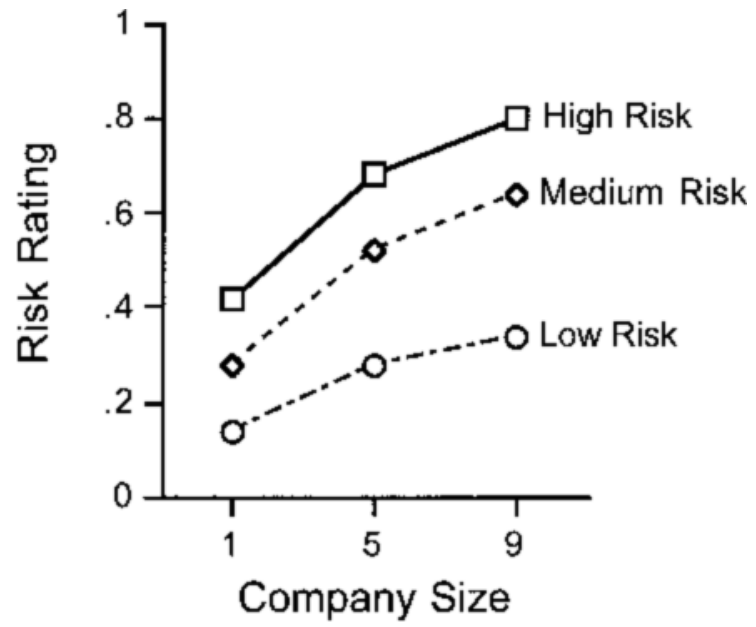

Figure 6. Simulated results of Experiment 2. The scale of the $y$ axis (0-1) reflects the fact that the output of the model is the correlation between the average stimulus representation and the prototype of an extrem ely high-risk employee. See the Appendix for details.

weighted in proportion to their similarity to a retrieval cue or prompt before averaging. In the present context, asking subjects to judge the risk of the typical employee might serve as a prompt that causes them to weight highrisk employees more heavily before averaging. Note, however, that simulations that incorporate this assumption in the most straightforward ways (described by Fiedler, 1996) fail to reproduce the present empirical results. The reason is that in the low-risk companies, the employees tend to be at uniformly low risk. Even if the highest risk employees are weighted particularly heavily, the effect of increasing the number of employees is still to decrease the similarity of the average employee to the highrisk prototype, resulting in lower risk judgments for the typical employee at a larger company. One promising alternative along these lines, however, is to give all the weight to the single employee that most closely resembles the high-risk prompt. In essence, this corresponds to Perloff and Fetzer's (1986) suggestion that people retrieve a single high-risk exemplar, which they use as a comparison. Because the highest risk employee in a larger group is likely to be at higher risk than the highest risk employee in a small group-regardless of the objective risk level of the company-this variant of the basic model also reproduces the group size effect described here.

Not only does the basic model provide a good first approximation of the empirical results reported here, but it also immediately suggests a number of additional hypotheses to test. For example, if selective encoding is really responsible for the group size effect, then encouraging subjects to encode low-risk cue values, in addition to highrisk cue values, should affect their risk judgments. For high-risk groups, the results should be essentially the same as those reported here. For low-risk groups, however, the typical member of a large group should be judged to be at lower risk than the typical member of a small group. If selective retrieval is partly responsible for the group size effect, then encouraging subjects to retrieve low-risk cue values or low-risk stimulus observations should affect their risk judgments. For example, asking subjects to judge the health rather than the risk of the typical employee might cause them to weight low-risk cue values or lowrisk employees more heavily than high-risk cue values or employees at the time of judgment. This might result in the typical employee at a larger company being judged in better health-yet at higher risk-than the typical employee at a smaller company (see Downs \& Shafir, 1999, for a conceptually similar result).

A final advantage of the BIAS framework is that, if necessary, it can also be used to model true motivational effects on people's personal risk judgments. For example, perhaps people tend to weight low-risk cue values more heavily when making judgments about themselves but tend to weight high-risk cue values more heavily when making judgments about their peers (Perloff \& Fetzer, 1986; Price et al., 1999; Weinstein, 1980). Such a differential weighting scheme could easily be introduced within the BIAS framework-much as differential weighting was included in the model presented here-and its implications could then be explored and tested.

\section{REFERENCES}

Alicke, M. D., Klotz, M. L., Breitenbecher, D. L., Yurak, T. J., \& VRedenburg, D. S. (1995). Personal contact, individuation, and the better-than-average effect. Journal of Personality \& Social Psychology, 68, 804-825.

American Heart Association (1998, November). AHA health risk awareness. Available at: http://www.ambrt.org/risk/quiz.html

BAKER, L. A., \& EMERY, R. E. (1993). When every relationship is above average: Perceptions and expectations of divorce at the time of marriage. Law \& Human Behavior, 17, 439-450.

Bauman, L. J., \& Siegel, K. (1987). Misperception among gay men of the risk for AIDS associated with their sexual behavior. Journal of Applied Social Psychology, 17, 329-350.

Boney-McCoy, S., Gibbons, F. X., \& Gerrard, M. (1999). Self-esteem, compensatory self-enhancement, and the consideration of health risk. Personality \& Social Psychology Bulletin, 25, 954-965.

Dougherty, M. R. P., Gettys, C. F., \& Ogden, E. E. (1999). MINERVA-DM: A memory processes model for judgments of likelihood. Psychological Review, 106, 180-209.

Downs, J. S., \& SHAFIR, E. (1999). Why some are perceived as more confident and more insecure, more reckless and more cautious, more trusting and more suspicious, than others: Enriched and impoverished options in social judgment. Psychonomic Bulletin \& Review, 4, 598-610.

FIEDLER, K. (1996). Explaining and simulating judgment biases as an aggregation phenomenon in probabilistic, multiple-cue environments. Psychological Review, 103, 193-214.

Hamilton, D. L., \& Gifford, R. K. (1976). Illusory correlation in interpersonal perception: A cognitive basis of stereotype judgments. Journal of Experimental Social Psychology, 12, 392-407.

HARRIS, P., \& MidDLETON, W. (1994). The illusion of control and optimism about health: On being less at risk but no more in control than others. British Journal of Social Psychology, 33, 369-386.

HinTZMAN, D. L. (1986). "Schema abstraction" in a multiple-trace memory model. Psychological Review, 93, 411-428.

Hintzman, D. L. (1988). Judgments of frequency and recognition memory in a multiple-trace memory model. Psychological Review, $\mathbf{4}$, 528-551. 
Janz, N. K., \& Becker, M. H. (1984). The health-belief model: A decade later. Health Education Quarterly, 11, 1-47.

Klar, Y., MedDing, A., \& SAREl, D. (1996). Nonunique invulnerability: Singular versus distributional probabilities and unrealistic optimism in comparative risk judgments. Organizational Behavior \& Human Decision Processes, 67, 229-245.

Klein, W. M., \& WeInstein, N. D. (1997). Social comparison and unrealistic optimism about personal risk. In B. P. Buunk \& F. X. Gibbons (Eds.), Health, coping, and well-being: Perspectives from social comparison theory (pp. 25-61). Mahwah, NJ: Erlbaum.

McKenna, F. P. (1993). It won't happen to me: Unrealistic optimism or illusion of control? British Journal of Psychology, 84, 39-50.

Middleton, W., Harris, P., \& Surman, M. (1996). Give 'em enough rope: Perception of health and safety risks in bungee jumpers. Journal of Social \& Clinical Psychology, 15, 68-79.

Perloff, L. S., \& Fetzer, B. K. (1986). Self-other judgments and vulnerability to victimization. Journal of Personality \& Social Psychology, 50, 502-510.

Peterson, C. R, \& BeAch, L. R. (1967). Man as an intuitive statistician. Psychological Bulletin, 68, 29-46.

Price, P. C., Lee., M., \& Voth, R. (1999, April). Computer simulation of unrealistic optimism in personal risk judgments. Paper presented at the meeting of the Western Psychological Association, Irvine, CA.

Regan, P. C., SNyder, M., \& Kassin, S. M. (1995). Unrealistic optimism: Self-enhancement or person positivity? Personality \& Social Psychology Bulletin, 21, 1073-1082.

Sмiтн, E. R. (1991). Illusory correlation in a simulated exemplar-based memory. Journal of Experimental Social Psychology, 27, 107-123.

Sмith, E. R., \& Zaraté, M. A. (1992). Exemplar-based model of social judgment. Psychological Review, 99, 3-21.

Svenson, O. (1980). Are we all less risky and more skillful than our fellow drivers? Acta Psychologica, 47, 143-148.

TAY LOR, S. E. (1989). Positive illusions: Creative self-deception and the healthy mind. New York: Basic Books.

WEINSTEIN, N. D. (1980). Unrealistic optimism about future life events. Journal of Personality \& Social Psychology, 39, 806-820.

Weinstein, N. D., \& KLeIn, W. M. (1996). Unrealistic optimism: Present and future. Journal of Social \& Clinical Psychology, 15, 1-8.

Whitley, B. E., \& Hern, A. L. (1991). Perceptions of vulnerability to pregnancy and the use of effective contraception. Personality \& Social Psychology Bulletin, 17, 104-110.

\section{APPENDIX \\ BIAS Simulation of Experiment 2}

The prototype of an extremely high-risk employee was represented as an eight-element vector, with each element representing one of the eight risk factors. For the first four risk factors, a value of +1 represented the high-risk value and a value of -1 represented the low-risk value. For the second four risk factors, a value of -1 represented the high-risk value and a value of +1 represented the low-risk value. For each company, a set of $N(1,5$, or 9$)$ stimuli was created by inverting (multiplying by -1 ) each of the high-risk cue values of the prototype with inversion probability, $i$. This probability was set to .75 for the lowrisk companies, .50 for the medium-risk companies, and .25 for the high-risk companies. To simulate the selective encoding of cue values, each cue value was then inverted with loss probability, $l$. This probability was set to .25 for high-risk values and .50 for low-risk values. This preserved most of the original stimulus information about the high-risk cue values and lost essentially all of the original information about the low-risk cue values. Note that selective encoding could also be simulated by multiplying the cue values by 0 with probability $l$ (Dougherty et al., 1999; Hintzman, 1988), but the results of the simulation are affected very little by this choice. The judgment of the risk of the typical employee was made by first computing the mean of each of the eight risk factors across the $N$ representations, and then computing the correlation between the resulting eightelement vector and the eight-elementhigh-risk prototype vector. A higher correlation, therefore, represented a higher risk judgment. This process was repeated 250 times, simulating the judgments of 250 subjects, for each of the nine unique combinations of objective risk and company size. It is the means of these 250 simulated judgments that are presented in Figure 5.

(Manuscript received July 20, 1999; revision accepted for publication September 8, 2000.) 